\section{Defining Cancer Care Quality or Delivering Quality Cancer Care?}

Matt Brow; J. Russell Hoverman, MD; Debra Patt, MD; Bill Herman;

Diana Verrilli; Jody Garey, PharmD; and Roy Beveridge, MD

Many in the oncology world see as accepted that quality and value are difficult to define and that no specialty-wide consensus is likely to be reached in the short term about how to measure and report success at improving either. Others find the definition just beyond their grasp and suggest that, like pornography, they know it when they see it. Large organizations, publications, conferences, and processes have sprung up around the concept, and various attempts have been made to develop "measures of quality" that can characterize a practice or physician in this area. This work includes ongoing efforts within and among the National Quality Forum, NCCN, ASCO, The US Oncology Network (The Network), the Community Oncology Alliance, and Ontario Cancer Care to identify quality measures - many of which focus on surrogate processes or activities that should lead to quality.

Instead, however, we believe this effort and investment should focus on outcomebased measures, and we offer a paradigm for measuring interventions:

- Quality is the efficient delivery of evidence-based care by trained clinicians in an accessible setting.

- In this context, value is providing higher quality care at the same cost, or the same quality at a lower cost.

We believe these concepts are at the core of cancer care delivery, whether in the community or academic setting and across specialties, including medical oncology, radiation oncology, gynecologic oncology, urology, and surgery. Achieving quality will require integrated, coordinated care with clinical teams spanning these professionals and settings. It requires not only coordination among clinicians of different specialties but also investment in infrastructure resources to facilitate the integration of process development and clinical decision support tools that support evidence-based practices that optimize practice efficiency and care delivery.

If the right investments have been made and communications highways created, these definitions are measurable, reportable, and comparable (without significant manual effort). Just as important, these definitions allow quality and value to evolve over time as the evidence evolves. Consequently, the highest-quality treatment pathways, and even the best modalities of treatment, may be vastly different in the future. For example, advance care planning and palliative care services would likely not have been included in the concept of evidence-based care 10 years ago. Today, however, the data suggest that palliative care concurrent with disease-directed care can improve outcomes and patient experiences while reducing costs. This view is also supported by a decade of hypothesis, consensus-building, technology investment, data collection, and analysis by physicians and supporting clinicians and staff of The Network, who we believe pioneered the concepts of narrowly drawn, evidence-based pathways for care in oncology.

Nearly a decade ago, physicians in The Network decided to develop Level I Pathways, evidence-based guidelines that redirect the wide range of treatments in oncology care into more precise, clinically proven treatment options. (More information is available at http://www.usoncology.com/cancercareadvocates/ AdvancingCancerCare/DeliverHigh-QualityCare/LevelIPathways.) The Level I Pathways program helps physicians eliminate disparities in care by refining potential

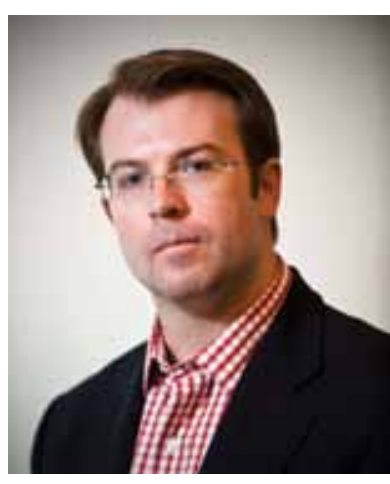

\section{Matt Brow}

Matt Brow is Vice President, Public Policy \& Reimbursement Strategy, McKesson Specialty Health \& The US Oncology Network.

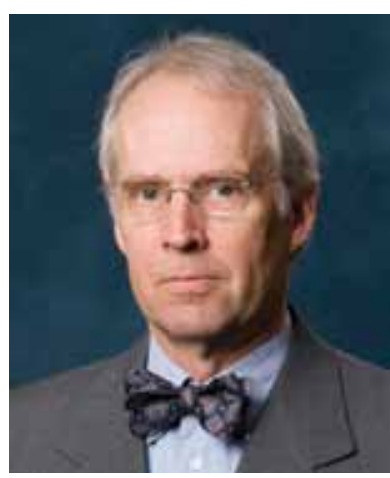

J. Russell Hoverman, MD J. Russell Hoverman, MD, is Shareholder, Texas Oncology; Medical Director, Managed Care, The US Oncology Network.

The ideas and viewpoints expressed in this editorial are those of the author and do not necessarily represent any policy, position, or program of NCCN. 


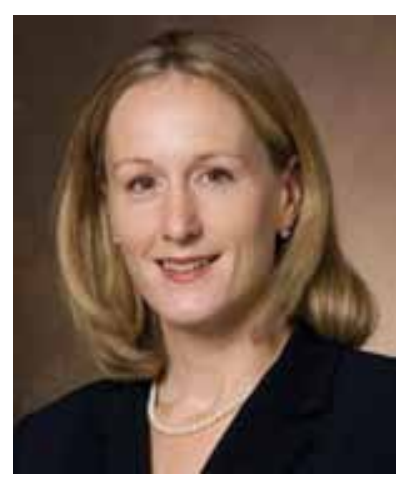

Debra Patt, MD

Debra Patt, MD, is Shareholder, Texas Oncology; Medical Director, Clinical Pathways, The US Oncology Network.

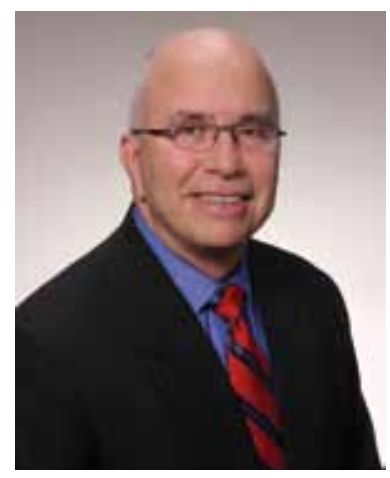

Bill Herman

Bill Herman is Senior Vice President, Practice Services, McKesson Specialty Health \& The US Oncology Network. choices to those with proven clinical effectiveness and acceptable toxicity, moving beyond broad label indications and public-domain guidelines. The program facilitates evidence-based choices in an efficient way at the point of decision-making in clinical care, and incorporates a supportive reporting process. When data support a treatment regimen as having superior efficacy and safety end points, that course of therapy is the suggested choice of treatment. When no clear clinical superiority exists, costs are then factored into the Pathway decision as an attempt to maximize value. Level I Pathways strive to use level 1 evidence, but other types of data may be used when level 1 evidence is not available.

Level I Pathways are developed and maintained by the Pathways Task Force (PTF), a subcommittee of the US Oncology Network Pharmacy \& Therapeutics (P\&T) Committee, which represents more than 1000 practicing community oncologists in The Network. PTF voting members consist of the PTF chairman, the P\&T chairman, and a physician chairman for each respective pathways area. Pathways areas include solid tumors, hematologic diseases, supportive care, advance care planning, radiation, and imaging. Disease subcommittees consisting of additional experts also participate in making recommendations. The PTF is supported by a team of pharmacists and data analysts who provide supporting data, implement clinical decisions into the electronic technology platform, and ultimately provide reports based on performance.

Development and maintenance of Level I Pathways is an ongoing process of collecting and analyzing key findings from medical journals, scientific meetings, clinical experience, and patient outcomes. Participating physicians and pharmacists are "firewalled" from commercial activities, and conflict of interest and nondisclosure agreements are maintained on an annual basis. Established Level I Pathways are reviewed at least annually, and may be reviewed more often, depending on the emergence of new data. Reviews occur via a quarterly process that lasts about 6 months from the time of evidence review to launch. Building new pathways occurs separately and may take longer depending on the complexity of the disease. Each Level I Pathway undergoes further review and update by a research committee of oncologists who are experts in the disease. Level I Pathway proposals are disseminated to the entire network for review and comments during an "open comment" period, with final review and approval by the P\&T committee.

Although physicians and practices in The Network are not required to adhere to Pathways, many have payer contracts that incentivize adherence. Each practice determines the process for implementing Pathways into individual workflow; however, centralized resources and programs that are further customized to address each practice's needs are also available. The specific areas of focus for reporting are data collection, pathway adherence, and exceptions (reasons for not using a recommended pathway choice). Because a pathway or guideline cannot address every clinical or patient-specific scenario, Pathways try to address $80 \%$ of patient care situations, with the realization that off-pathway regimens are acceptable in certain scenarios.

The initiative began with the supposition that such an approach would lead to cost-effectiveness, along with quality. However, at the time there were no data, and not all agreed with the premise. Skeptics within The Network, and within the payer community, argued that adherence to evidence-based pathways might have the offsetting effect of increasing overall costs. Adherence, they suggested, would increase the cost for those who traditionally spend less by as much, or more, than it would bring down the cost for those who traditionally spend more. The net result, according to this argument, would be higher overall costs.

What has evolved, however, is a culture change. Physician-generated pathways drive engagement and facilitate a culture of quality and value-based care. Because this culture is accompanied by investment and engineering in clinical decision support 
tools embedded within the electronic health record, use optimizes practice efficiency. Without these, workflow and patient care are obstructed.

In addition to developing an efficacious process, we needed to evaluate our outcomes. A joint study with Aetna evaluated the cost-effectiveness of Level I Pathways for patients with non-small cell lung cancer in the community setting. ${ }^{1}$ This study found that certain outpatient costs were $35 \%$ lower for patients treated according to Level I Pathways, while maintaining equivalent health outcomes. Similarly, a joint study with Milliman found that evidence-based care for patients with colon cancer results in significant cost savings with equivalent health outcomes. ${ }^{2}$ The study showed a total cost savings of more than $30 \%$, or $\$ 53,000$, for the adjuvant treatment of colon cancer and $\$ 60,000$ for the first-line treatment of metastatic colon cancer.

These studies prove that adherence and cost are measurable and reportable, but our practical experience has shown that this part of the equation can be far more complex than defining the superior pathway in a condition for which 100 different protocols were used. Although difficult, however, this aspect is critical to success in pathways adoption and adherence. Our experience is that physicians frequently believe themselves to be practicing evidence-based medicine or treating within established guidelines, but the true story emerges only with automated review.

Another concern is that building an electronic health record (EHR) around the ability to deliver decision support (and your adopted pathway for any given instance) when it really matters-at the point of decision — can be expensive. Agreement on common definitions can also be difficult, even for elements as simple as what constitutes third-line or adjuvant therapy. Perhaps the most difficult task, however, is accessing the whole picture of what happens to your patient outside of the cancer center and how much it all costs.

Health care is in an era of dynamism, perhaps more rapid than any we have seen. As the landscape evolves, change will come either from within or from outside our systems of care. Increasing pressures on government, employers, insurers, and individuals are demanding rapid change, and there are many out there who would substitute their judgments for physicians' to cut costs. We prefer to lead the change. This speaks to an overarching need to adopt a technology platform that provides a continuously updated source of data to inform decision making and to feed data back into the loop as physicians and patients make clinical choices and the outcomes associated with those choices are revealed. Such a structure becomes increasingly vital as personalized medicine leads to myriad new options focused on an individual's genetic and disease characteristics.

We believe that this environment requires all oncologists to stand up and be counted by living the principles they hold dear. For clinicians who agree that the core of oncology quality and value is care based on evidence, now is the time to:

- invest in, or fine-tune, systems that allow point-of-decision support within the EHR and facilitate real-time reporting on adherence to pathways that allow practices to connect with payers;

- adopt a narrow set of physician-driven, evidence-based pathways that have undergone rigorous review, are continuously updated as the evidence evolves, and are easily incorporated into the EHR;

- place patients at the center of the decision process so that the pathways are flexible enough to allow for evidence-based variations to meet specific patient needs;

- use the EHR to track and report adherence to the pathways adopted; and

- connect in partnership with payers to better understand and measure the costs of care.

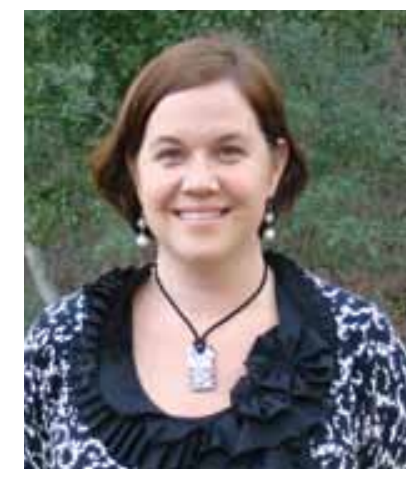

Diana Verrilli

Diana Verrilli is Vice President \& General Manager, Payer \& Revenue Cycle Services, McKesson Specialty Health \& The US Oncology Network.

Jody Garey, PharmD

Jody Garey, PharmD, supports the Level I Pathways program as a Clinical Pharmacist for McKesson Specialty Health \& The US Oncology Network. 
February 2013

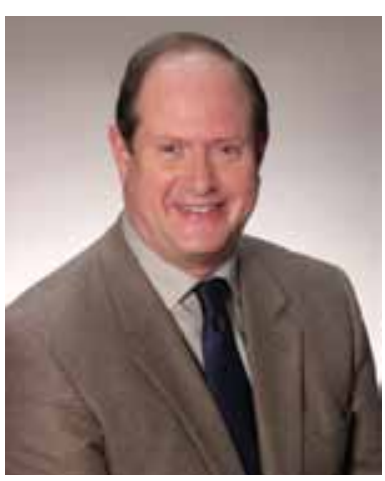

Roy Beveridge, MD

Roy Beveridge, MD, is Chief Medical Officer, McKesson Specialty Health \& The US Oncology Network.
However, even when these necessary initial steps are taken, a significant final step remains: negotiating payment arrangements that reward this type of care and using electronic information systems that connect to the payers' systems to automatically approve (and exempt from prior authorization, precertification, prepayment review) the approaches agreed upon. The rewards may be in the form of favorable reimbursement rates, exemption from administrative requirements such as prior authorization, or even exclusive or preferred relationships between payers and high-value providers.

However quality and value are defined, oncologists-both community and academic — and payers— both private and government—-must work together to drive improvements in the quality and cost of cancer care to create a sustainable future. With the rise of consumer power and the continuing increase in potential costs of care, the successful cancer-care provider must focus on evidence-based quality care, lowering the overall costs of care (optimizing value), and improving the patient experience. At the same time, payers and the government must be able to recognize and reward high-quality, cost-effective care if they want it to flourish. We continue to believe that physicians with sophisticated information technology solutions and who manage the care of a patient population and oversee the care experience will be the key lever point for success by defining high-quality, high-value care and by insisting on appropriate payment for that care.

\section{References}

1. Neubauer MA, Hoverman JR, Kolodziej M, et al. Cost effectiveness of evidence-based treatment guidelines for the treatment of non-small-cell lung cancer in the community setting. J Oncol Pract 2010;6:12-18.

2. Hoverman JR, Cartwright TH, Patt DA, et al. Pathways, outcomes, and costs in colon cancer: retrospective evaluations in two distinct databases. J Oncol Pract 2010;7(Suppl 3):52s-59s.

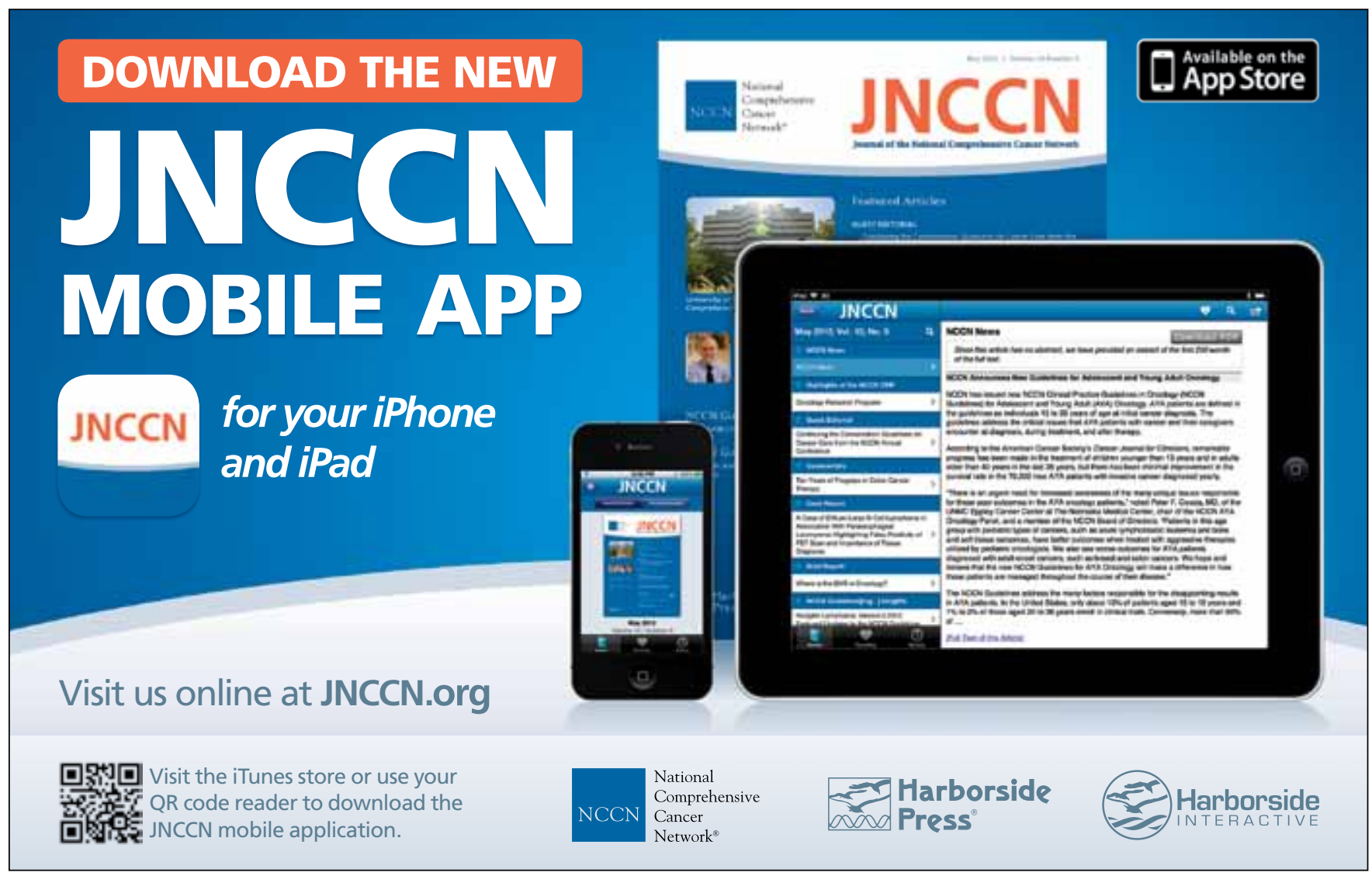

